SC-MAG 408

LBL33002

\title{
SSC Quadrupole Magnet Performance at LBL
}

A. F. Lietzke, P. Barale, R. Benjegerdes, S. Caspi, J. Cortella, D. Dell'Orco, W. Gilbert, M.I. Green, K. Mirk, C. Peters, R. Scanlan, C.E. Taylor, and A. Wandesforde

Superconducting Magnet Group

Lawrence Berkeley Laboratory

Berkeley, CA 94720

\section{INTRODUCTION}

LBL contracted to design, construct, and test four short $(1 \mathrm{~m})$ models and six full-size $(5 \mathrm{~m})$ models of the Superconducting Super Collider (SSC) main-ring 5 meter focusing quadrupole magnet ( $211 \mathrm{Tesla} / \mathrm{meter}$ ). The training performance of these magnets are herein summarized.

\section{MAGNET CONSTRUCTION}

Each magnet contained eight coils arranged in a two-layer "cos $2 \theta$ " pattern around a circular $(40 \mathrm{~mm})$ bore (Figure 1). Coil construction and magnet assembly details can be found elsewhere[1,2,6]. In summary, all coils were wound under tension with 30-strand NbTi cable, and insulated with Kapton and epoxy-impregnated fiber-glass cloth. The coils were compressed to final size and heated enough to polymerize the epoxy. The resulting rigid coils were assembled onto an alignment mandrel and compressed by several quadrupole-symmetric, interlocking aluminum or S.S. collar-plate assemblies. The collar-packs were aligned by four collar tabs which fit into key-way grooves machined into the iron "yoke-blocks". Structural analysis of this collaring system was done by D. Dell'Orco [3]. Each iron yoke-block was aligned by inserting keys through openings in the shell. Weld shrinkage from the two full-length axial welds locked this alignment to the shell. An end-to-end twist of less than $3 \mathrm{~m}$ radians was achieved for all $5 \mathrm{~m}$ magnets. The end-regions, outside the iron flux-return, were compressed azimuthally by an aluminum collet during the collaring procedure (QSC401 \& 402, used S.S. C-shells.) Each end was immobilized and compressed axially before testing. Construction extended over a period of two years. Changes were made to the magnet end-region clamping and immobilization systems, collaring material, collaring pressure, collaring mandrel length and hardness, collar/yoke friction, conductor manufacturer and curing \& collaring procedures.

\section{TEST PROCEDURES AND RESULTS}

The test procedures are discussed in detail elsewhere [6]. Magnets were tested in a horizontal boiling helium $(1 \mathrm{Atm})$ cryostat. The magnetic, strain-gage and training responses to two thermal cycles were measured. The quadrupole gradient, and relative multipole purity were determined from Fourier analysis of the rotating coil signals $[4,6]$. Magnetic and strain-gage measurements were taken on-the-fly. The voltage-tap data was analyzed to determine quench-origin and propagation characteristics. Quench-training proceeded at $4.3 \mathrm{~K}$ until a plateau was achieved or subcooling $(2.5 \mathrm{~K})$ was used to accelerate the training process. The early short $(1 \mathrm{~m})$ magnets were also trained at $1.8 \mathrm{~K}$ (10kA) to help identify potential weak areas. The MIITs [6] were calculated to compare various magnet protection methods.

Ramp-rate sensitivity (tested from $1 \mathrm{~A} / \mathrm{s}$ to $10 \mathrm{kA} / \mathrm{s}$ ) varied from magnet ot magnet (Figure 2). The low ramprate sensitive magnets had either been collared more than once (QSC401, QCC405) or used a new conductor surface treatment (QCC406). These magnets also exhibited higher maximum MIITs [6] ( $<9$ MIITs, unprotected).

Figure 3 shows the $4.3 \mathrm{~K}, 16 \mathrm{~A} / \mathrm{s}$ training behavior of these magnets. All training quenches started in the poleturn, usually in the inner coil at the start of training, but moving to the outer coil (as expected [1]) when the plateau was achieved. Long (5 meter) magnets (Figure 4a) trained as fast as short (1 meter) magnets [6]. 


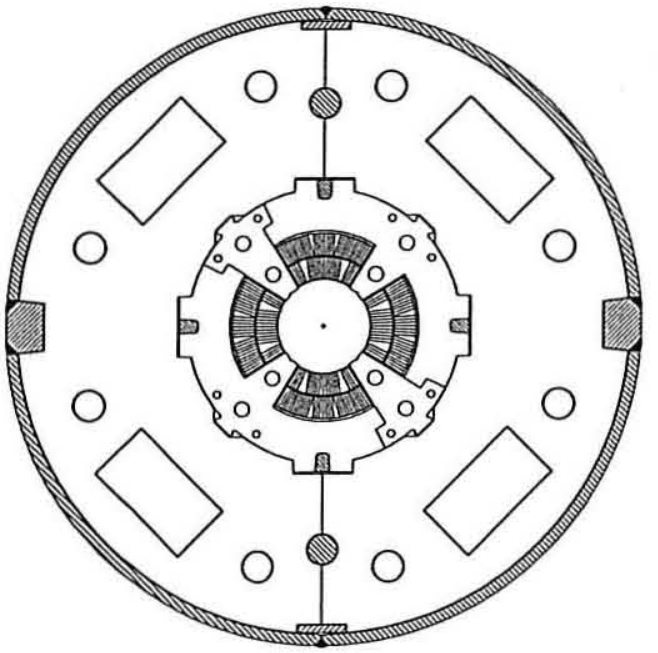

Figure 1. Collared Coil cross-section: coils, pole-pieces, collars, keys and yoke alignment tabs
RAMP RATE SENSITIVITY

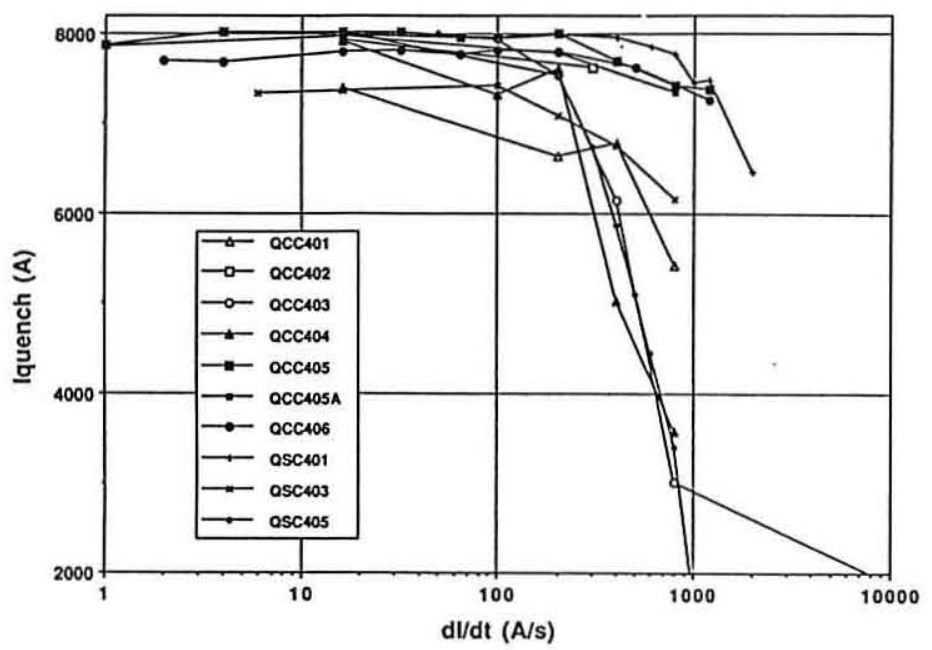

Figure 2. Ramp-rate Dependence of Plateau Current (all Magnets)

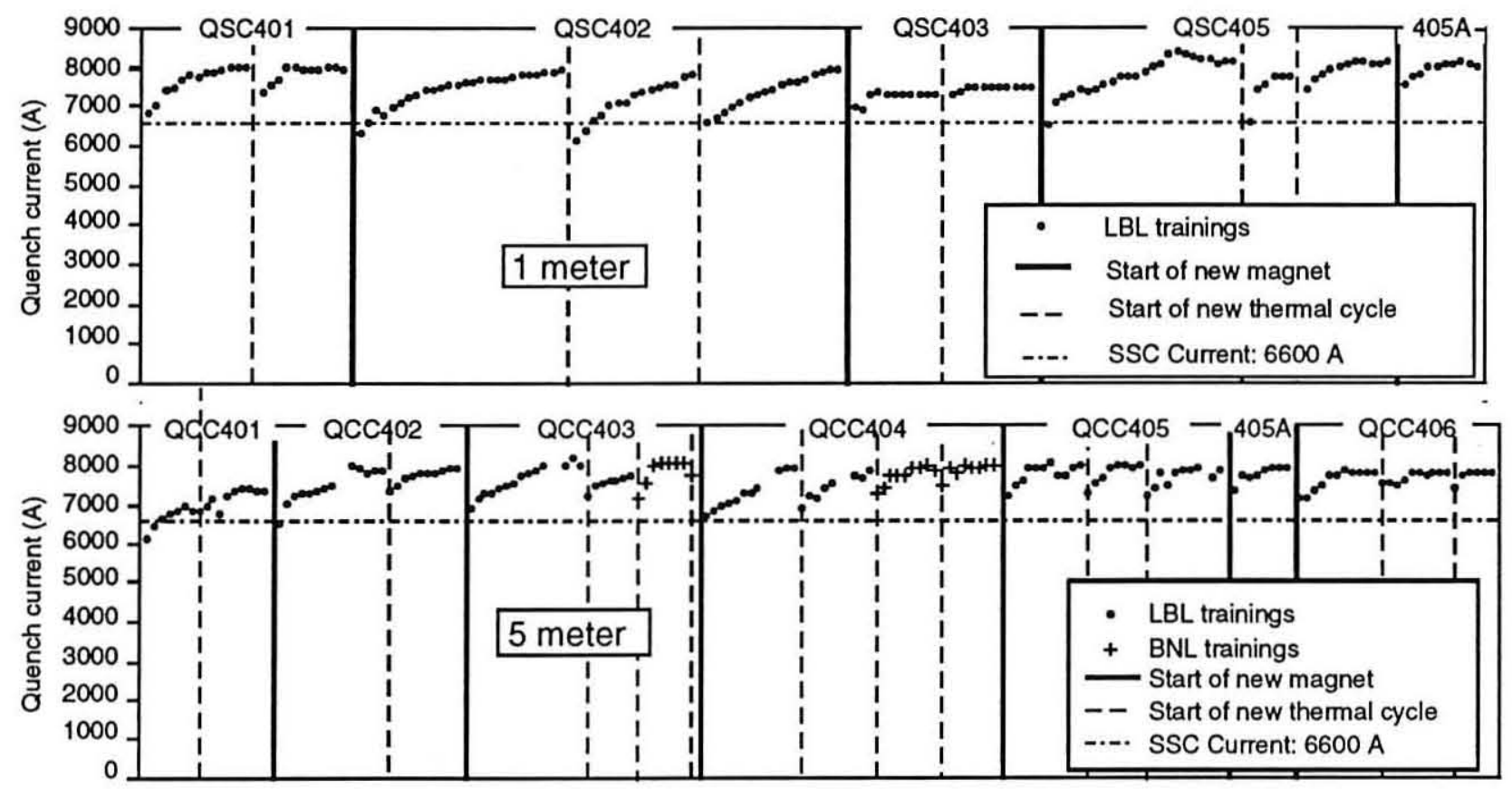

Figure 3. Quadrupole Training Records (16A/s, 4.3K)

Performance improved substantially between the first and last magnets. The last two magnets required no training below 7170A. Later magnets generally started at a higher current (except QCC 404 and QCC 406) and required fewer quenches to achieve and maintain plateau levels. Magnets that had lower plateau currents required fewer quenches to attain plateau: QSC403, QCC406. Sub-cooling $(<2.5 \mathrm{~K})$ reduced the number of quenches needed to reach the plateau current.

All magnets had to be retrained (Figure $4 \mathrm{~b}$ ) after being warmed to room temperature. Subsequent thermal cycles usually started higher and trained faster (some more dramatically than others). Clamping the collared coil firmly in the yoke did not significantly change the retraining (compare QCC405A with QCC405, Figure 4b).

Quench-origins (Q-O's): All training quenches originated in the pole turn (shown in Figures 5a,b, relative to voltage taps, strain-gages, collar-pack boundaries, hard-mandrel edges, and the inter-layer ramp-splice). 


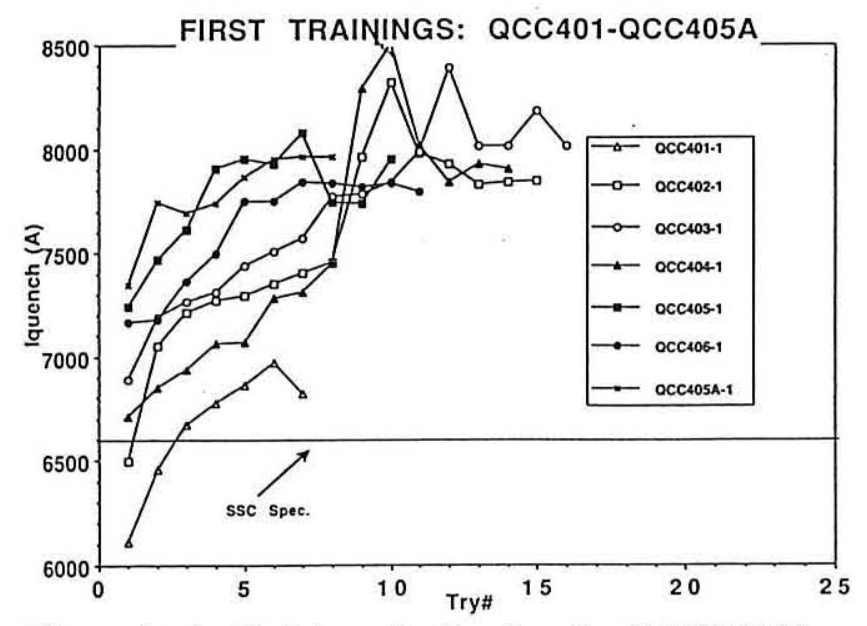

Figure 4a. 1st Trainings: 5m Quadrupoles (4.3K,16A/s)

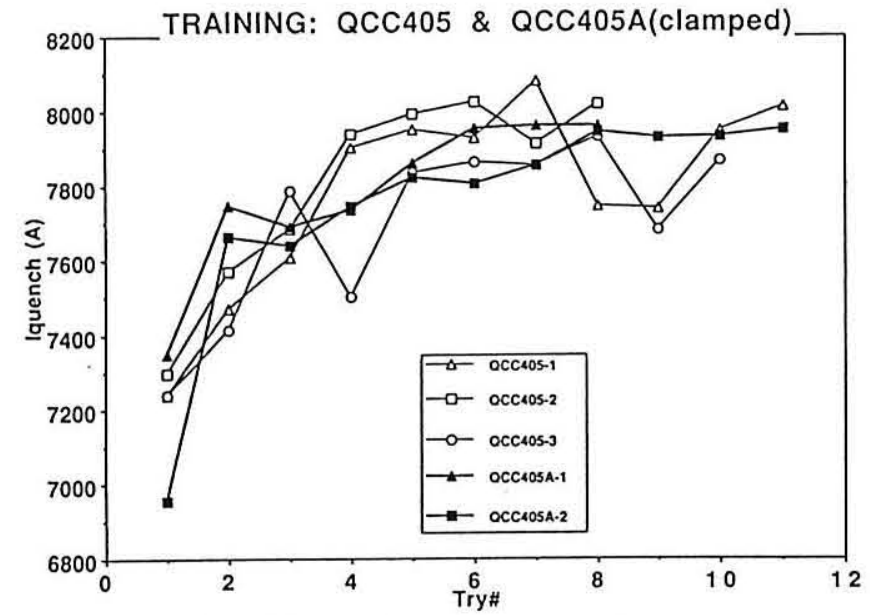

Figure 4b. Re-Training after clamping \& thermal cycling $(4.3 \mathrm{~K}, 16 \mathrm{~A} / \mathrm{s})$

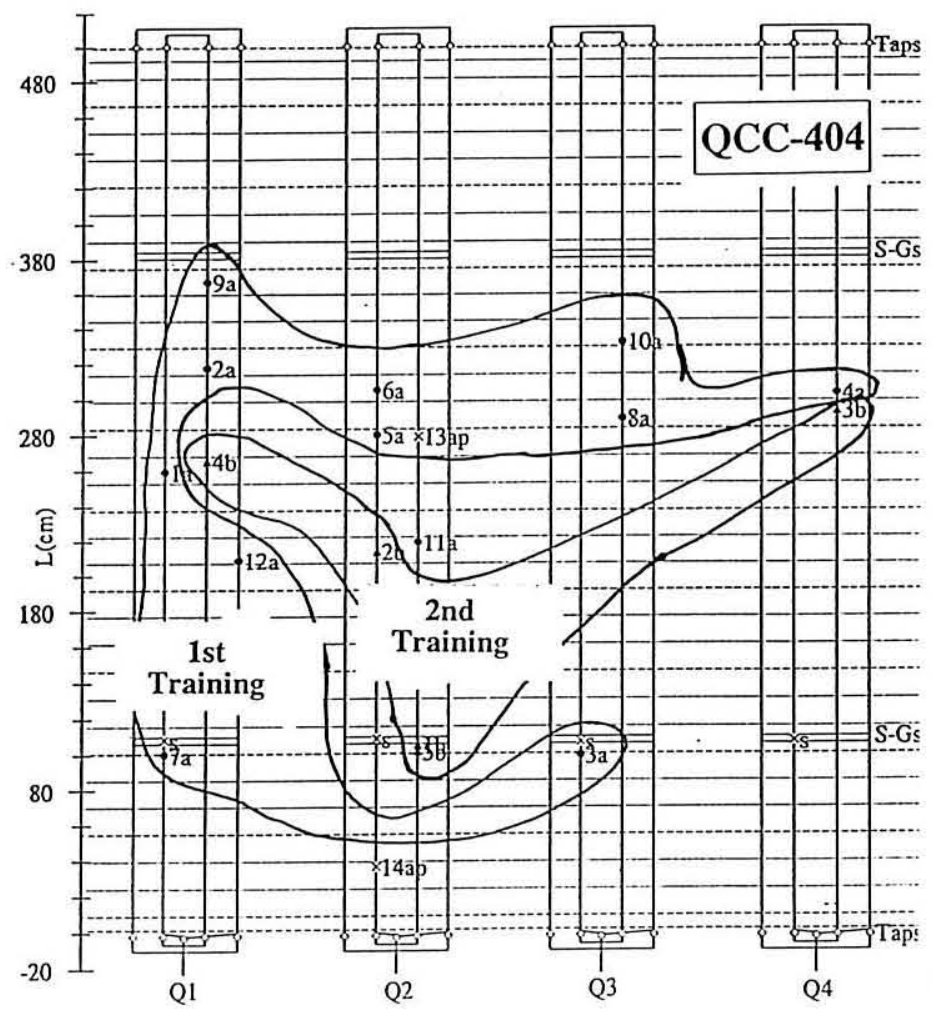

Figure 5a. Quench-Origins: QCC404 Trainings

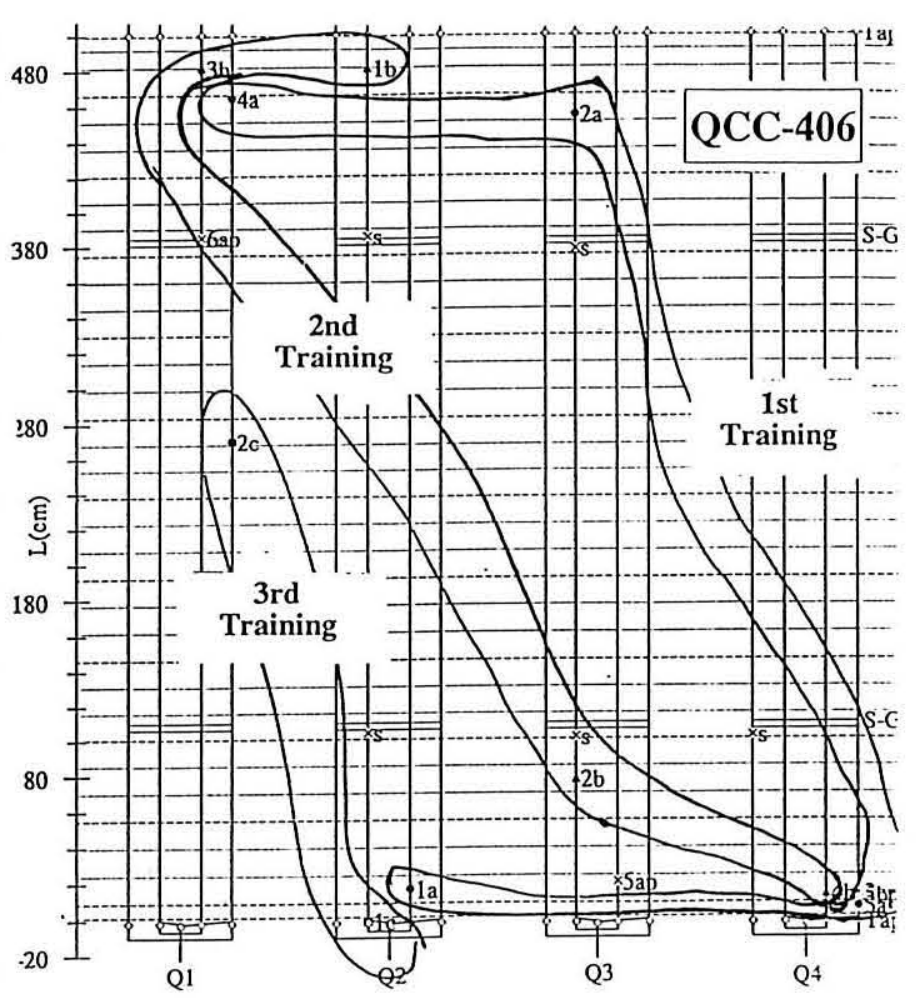

Figure 5b. Quench-Origins: QCC 406 Trainings

Each magnet showed a characteristic pattern of quench-origins (Q-O's). QCC404 (Figure 5a) revealed a band of Q-O's near the middle, and QCC406 (Figure 5b) suffered most of its Q-O's near its ends. Axial nonuniformities were usually preserved over thermal cycles but usually with a change in quadrant. Many Q-O's were at, or near, collar-pack boundaries. Strain-gage-triggered quenches $(\mathrm{x})$ were used to estimate systematic and random errors. A magnet rarely repeated any particular quench-origin. Exceptions QSC401 and QSC402 were later discovered to have end-clamp design oversights [6], and QCC406 is believed to have had a broken strand (right corner, Figure 5b).

Strain-gage response: After training, most magnets produced a linear response to the Lorentz load [6]. In spite of considerable variations in the initial stress (correlated to the measured coil sizes), substantial pole pressures usually remained up to 8kA. QCC406, however, suffered an unexpected amount of creep. Some locations were 
unloaded above 6kA [6], yet no obvious effect on training was observed. Short models were also tested to 10kA @ $1.8 \mathrm{~K}$ with no lack of trainability. Ramping into virgin territory ( $>5 \mathrm{kA}$, Figure $6 \mathrm{a}$ ) revealed a steeper unloading rate (i.e., lower coil stiffness). This "mechanical training" characteristic repeated on subsequent thermal cycles and is suspected to be related to the manner in which these magnets also "forgot" their training. While ramping to 1 st quench, an anomalous stiffening was often observed prior to quenching (Figure 6b). The quench-origin was often located near the load cell which exhibited the largest anomaly. Magnetic multipole purity was very good [7].

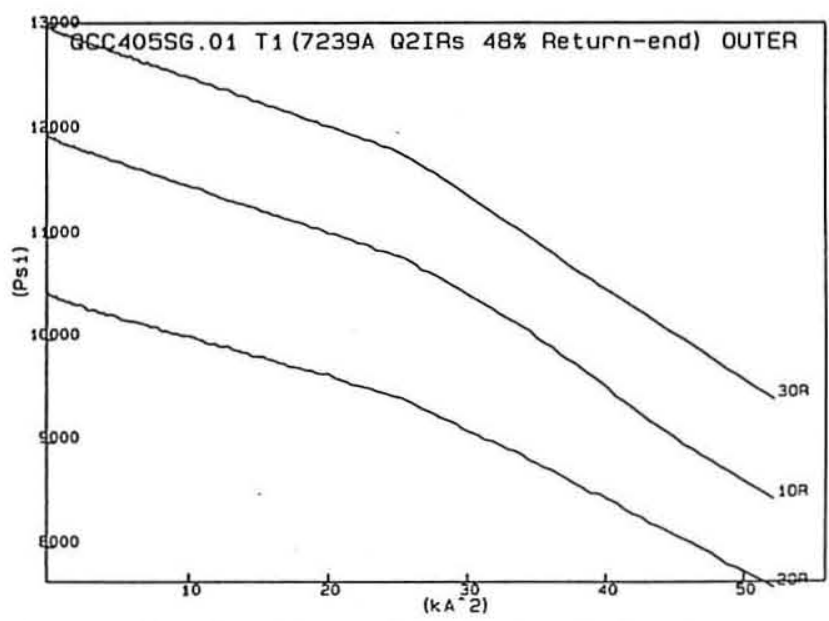

Figure 6a. Strain-Gage: Entering Virgin Territory

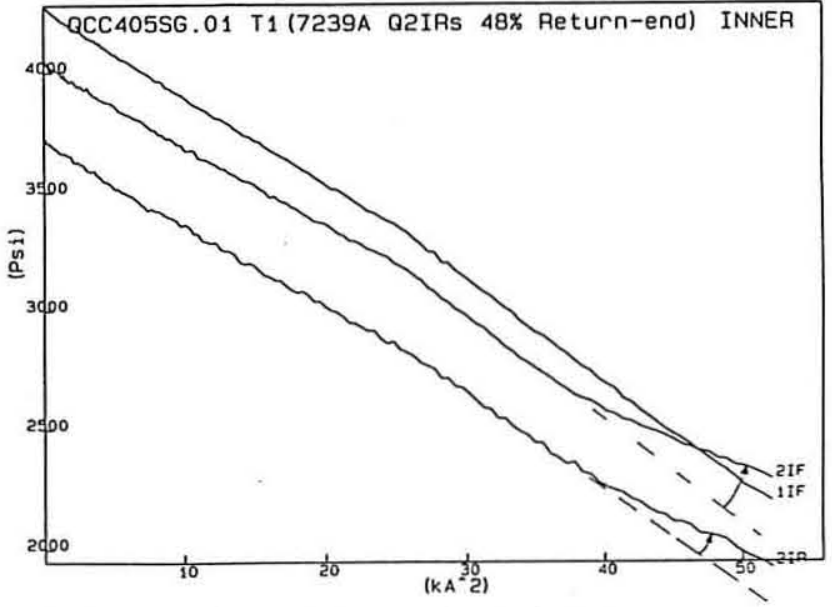

Figure 6b. Strain-Gage: Near 1st Quench

\section{DISCUSSION AND SUMMARY}

The importance of adequate coil clamping was dramatically supported by the improved training after the endregion cold-clamping pressure was retained (magnets after QSC402, where some repetitive quench-origins were eliminated). How much pressure is enough, is questionable in view of QCC406's unloading, yet second best training.

The issue of optimum yoke-collar friction is also unclear. QSC405A (re-collared, welded and clamped in the yoke) trained the fastest of all magnets. QCC404, on the other hand (lightly clamped), trained much more slowly than magnets built to slide (QCC403, 405, 406); and QCC405A (clamped QCC405) showed no improvement at all.

The larger compliance observed during the first excitation, suggests that the cable/collar system has some freedom to deform under Lorentz loading. Stiffening observed just prior to quenching suggests that some parts of the system resist this deformation, and may constitute a source of stored energy capable of triggering quenches. Lack of memory retention after thermal cycling suggests the existence of restoring forces that are strong enough to restore a somewhat "virgin" state during some part of the thermal cycle. Movement of the quench-origins to new locations implies that the magnet is "reset" to a different "virgin" condition.

The Miits/ramp-rate sensitivity correlation is evidence that dI/dt-triggered quenching can decrease the cable temperature.

Ten pre-prototype magnets including six 5m models were designed, constructed and tested at LBL for proofof-principle demonstrations of the design proposed to meet SSC operational requirements. Except for modest training above the anticipated SSC operating point, the magnets performed very well and proved to be self-protecting. Some design flaws were identified and corrected. The last two $1 \mathrm{~m}$ models and all the $5 \mathrm{~m}$ models have been reinstalled in cryostats at the SSC Laboratory, retested and used to achieve various milestones in their program.

[1] C. E. Taylor, et al; IEEE Trans. on Mag., vol. 27, No. 2, March 1991.

[2] S. Caspi, "The 40mm SSC Arc Quadrupole - Magnetic Design," SC-MAG-314, LBID-1677, November 1990.

[3] D. Dell'Orco, "Finite Element Analysis of the QC Quadrupole Magnet for the SSC", LBL-29600, October 1989.

[4] M. I. Green, et.al, "Measurements of Magnetization Multipoles in Four Centimeter Quadrupoles for the SSC," Cryogenic Engr. Conf., Huntsville, AL, June 11-14, 1991.

[5] J. M. Cortella, et.al, "Mech. Prop. of 5m SSC Quadrupoles", Proc. of ASC, Chicago, Aug. 1992, SC-MAG 401.

[6] A. F. Lietzke, et. al., "Quadrupole Magnets for the SSC", Proc. of ASC, Chicago, August 1992, SC-MAG400.

[7] P. J. Barale, et. al., "Magnetic Meas. of 5 Meter Quadrupoles at LBL", (paper IV-C-2, this conference.)

Work supported by the Director, Office of Energy Research, Office of High Energy and Nuclear Physics, High Energy Physics Division, U.S. Department of Energy, under Contract No. DE-AC03-76SF00098. 